\title{
On the Generalization of the Law of Cosines of Triangles to Three and More Dimensions
}

\author{
M.A. Murray-Lasso \\ Unidad de Enseñanza Auxiliada por Computadora \\ Departamento de Ingeniería de Sistemas. División de Estudios de Posgrado \\ Facultad de Ingeniería, UNAM
}

(recibido: marzo de 2002; aceptado: julio de 2002)

\begin{abstract}
The Law of Cosines for triangles is generalized for tetrahedrons and simplices in higher dimensions. The result is obtained by noting that three vectors which vectorially add to the zero vector, that is closing a vector polygon, can be arranged in an equation placing the negative of one equal to the sum of the other two. When each member of the equation is multiplied with a dot product by itself and the dot product of the two vectors is expressed in terms of the magnitudes of the vectors and the cosine of the angle between them the Law of Cosines is obtained. The same idea with more than three vectors yields an Extended Law of Cosines that contains additional square terms and additional terms with cosines. The extension to the tetrahedron is achieved by representing the areas of its faces with vectors orthogonal to them and noting that their sum is the zero vector. When the foregoing idea is applied to the closed polygon an Extended Law of Cosines appears. The process is extended to the higher dimensional counterparts of the tetrahedron (the simplex) and the proof of its validity is accomplished through mathematical induction on determinant expressions for the corresponding "volumes" (which for lines reduce to lengths, for triangles to areas and for four and more dimensions turn into hypervolumes.) Numerical examples are provided to clarify ideas. Finally the cross product of two vectors which exists only for three-dimensional spaces is generalized to a cross product of $n-1$ vectors in an n-dimensional space. This cross product has many of the properties of the standard cross product in tree dimensions and can be used for the calculation of hyperareas and hypervolumes.
\end{abstract}

Keywords: Law of Cosines, Tetrahedron, Simple Hyperarea, Hypervolume, n-Dimensional

\section{Resumen}

Se generaliza la Ley de los Cosenos para los triángulos en cuatro y más dimensiones a los tetraedros y simplejos. El resultado se logra notando que para tres vectores cuya suma vectorial es el vector cero, es decir, que al sumarlos se cierra el polígono vectorial; se puede escribir una ecuación en la cual en el primer miembro aparece el negativo de uno de los vectores y en el segundo miembro aparece la suma de los otros dos. Cuando cada miembro se multiplica con punto por sí mismo y el producto punto de dos vectores se expresa como el resultado de sus magnitudes y el coseno del ángulo entre ellos, se obtiene la Ley de los Cosenos. La misma idea con más de tres vectores desemboca en la Ley de los Cosenos Extendida que contiene términos cuadráticos y términos con cosenos adicionales. La extensión al tetraedro se logra representando las áreas de sus caras con vectores ortogonales a las mismas y notando que su suma es el vector cero. Cuando la idea descrita se aplica al polígono de vectores cerrado, aparece la Ley Extendida de los Cosenos. El proceso se extiende a las contrapartes del 
tetraedro en cuatro y más dimensiones (simplejos) y la demostración de su validez se logra por medio de la inducción matemática sobre expresiones con determinantes que corresponden a los volúmenes (que para líneas se reducen a longitudes, para triángulos a áreas y para simplejos en cuatro y más dimensiones se convierten en hipervolúmenes). Se proporcionan ejemplos numéricos para aclarar las ideas. Finalmente, se señala que se puede extender el concepto de producto cruz a $n-1$ vectores en espacios $n$-dimensionales y aplicarse al cálculo de hiperáreas e hipervolúmenes.

Descriptores: Ley de cosenos, tetraedro, simplejo, hiperárea, hipervolumen, n-dimensional

\section{Introduction}

One of the important theorems of plane geometry is the so called Law of Cosines that states that in any triangle of vertices and internal angles A, B, C and opposing sides of lengths $a, b, c$, such as the one shown in figure 1 , the following formula known as the Law of Cosines holds (Welchons et al., 1965)

$$
a^{2}=b^{2}+c^{2}-2 b c \cos \mathrm{A}
$$

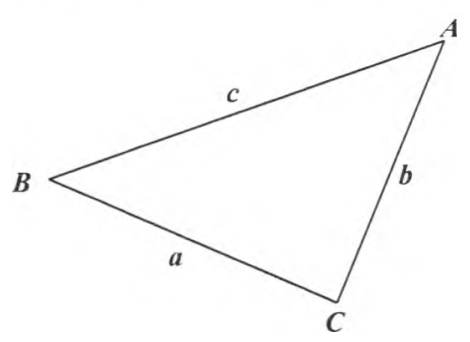

Figure 1

The formula is valid for any triangle labeled arbitrarily, as long as the labels on the sides are lower case letters corresponding to the capital letters used as labels for the oppossing angles. Note that when the angle at $\mathrm{A}$ is obtuse, $\cos \mathrm{A}$ is a negative number. The Pithagorean Theorem is a particular case of this more general formula, because in case the angle $\mathrm{A}$ is a right angle then $\cos \mathrm{A}=0$ and the expression reduces to the familiar

$$
a^{2}=b^{2}+c^{2}
$$

that is, the square of the hypothenuse is equal to the sum of the squares of the legs. In this article we extend the Law of Cosines to the tetrahedron and simplices in higher dimensions by establishing that triangles, tetrahedrons and higher dimensional simplices can all be represented by closed polygons of vectors in the corresponding spaces using the same strategy of proof used for the triangle.

\section{A Vector Proof of the Law of Cosines for the Triangle}

Although there are many ways to prove the Law of Cosines for the triangle, a particularly convenient method of proof which can be generalized to polygons in three and more dimensions uses vectors. In figure 2 , two of the sides of a triangle are represented by vectors $b$ and $c$.

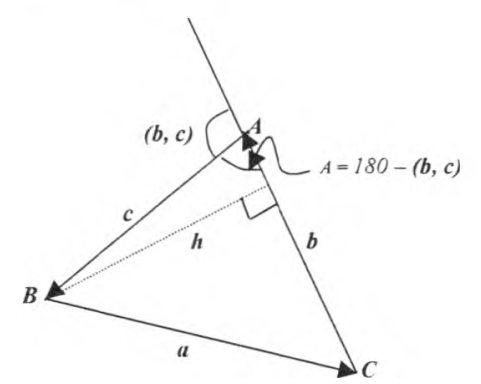

Figure 2

According to the triangle rule of vector adition with the senses chosen as shown in figure 2

$$
\boldsymbol{a}+\boldsymbol{b}+\boldsymbol{c}=\mathbf{0} \text { which can be written }-\boldsymbol{a}=\boldsymbol{b}+\boldsymbol{c}
$$

Now dot multiply both members of the last equation by themselves, we obtain

$$
a \cdot a=(b+c) \cdot(b+c)=b \cdot b+c \cdot c+2 b \cdot c
$$

this can be written

$$
|\boldsymbol{a}|^{2}=|\boldsymbol{b}|^{2}+|\boldsymbol{c}|^{2}+2|\boldsymbol{b}||\boldsymbol{c}| \cos (\boldsymbol{b}, \boldsymbol{c})
$$


where $(\boldsymbol{b}, \boldsymbol{c})$ is the angle between the vectors $\boldsymbol{b}$ and $c$ shown in figure 2 . To write the expression in terms of the internal angle $A$ at the vertex $\mathbf{A}$ as is usually done in elementary geometry, we note that $A=180$ $-(\boldsymbol{b}, \boldsymbol{c})$, hence $\cos (\boldsymbol{b}, \boldsymbol{c})=-\cos \mathrm{A}$ and we can write

$$
|\boldsymbol{a}|^{2}=|\boldsymbol{b}|^{2}+|\boldsymbol{c}|^{2}-2|\boldsymbol{b}||\boldsymbol{c}| \cos \mathrm{A}
$$

which is the Law of Cosines ordinarily written

$$
a^{2}=b^{2}+c^{2}-2 b c \cos \mathrm{A}
$$

where $a=|\boldsymbol{a}|, b=|\boldsymbol{b}|, c=|\boldsymbol{c}|$.

\section{Representation of Flat Areas by Vectors}

In order to easily extend the Law of Cosines to the tetrahedron through an analogy with what was done for the triangle, we will represent a flat area by means of a free vector whose direction is orthogonal to the plane of the area and whose sense is such that if we encircle the area inside a closed line traversed in a certain sense, the vector points in the direction in which a right screw would advance when turned in the same sense. This is a standard way of representing physical quantities acting on a plane through free vectors in many engineering applications (Seely et al., 1948; Cannon, 1967; Bueche, 1988; Hoffmann, 1966; Hague, 1939). The vector $\mathbf{V}$ that represents the area of the triangle of figure 2 can be written in terms of any two of the vectors that encircle it as follows:

$$
\begin{gathered}
\mathbf{V}=1 / 2(\boldsymbol{b} \times \boldsymbol{c})=1 / 2|\boldsymbol{b}||\boldsymbol{c}| \operatorname{sen}(\boldsymbol{b}, \boldsymbol{c})=1 / 2(\boldsymbol{a} \times \boldsymbol{b})= \\
1 / 2|\boldsymbol{a}||\boldsymbol{b}| \operatorname{sen}(\boldsymbol{a}, \boldsymbol{b}) \\
=1 / 2(\boldsymbol{c} \times \boldsymbol{a})=1 / 2|\boldsymbol{c}||\boldsymbol{a}| \operatorname{sen}(\boldsymbol{c}, \boldsymbol{a})
\end{gathered}
$$

since for the triangle of figure $2, h=|\boldsymbol{c}| \operatorname{sen}(\boldsymbol{b}, \boldsymbol{c})=|\boldsymbol{c}|$ $\operatorname{sen}[180-(\boldsymbol{b}, \boldsymbol{c})]$ is the height of the triangle considering that the vector $\boldsymbol{b}$ is the base. Similar considerations yield the other members of the equations.

\section{Choosing the Orientations of the Faces of the Tetrahedron}

Now consider the tetrahedron shown in figure 3 which is viewed from the top. Each triangular face is oriented in the way shown by the ellipses. The dotted circle corresponds to the triangular face upon which the tetrahedron rests on a horizontal plane. All faces of the tetrahedron are oriented so that the vectors representing them enter the tetrahedron as shown in the figure. The vector that represents the face upon which the tetraedron rests on a horizontal plane is directed directly towards the viewer and is drawn as a circle with a dot in the center to simulate the view of an arrow pointing directly towards the viewer.

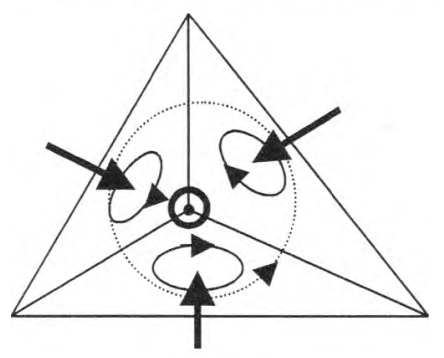

Figure 3

\section{The Law of Cosines for the Tetrahedron}

In order to establish the extension of the Law of Cosines to the tetrahedron we first prove that the four vectors representing its four faces when added according to the triangle law of vectors in three-dimensional space result in the zero vector, in other words, the four vectors when placed (in any order) one resting its tail on the tip of the arrow of the previous one while maintaining the direction and sense of each of the four arrows, the figure so formed is a closed polygon in three-dimensional space. We establish this algebraically using the fact that a vector that represents a triangle in three-dimensional space can be expressed as one half of the cross product of two of the vectors along two of the sides of the triangle. In figure 4 we show the tetrahedron of figure 3 with the same orientation for the faces and with the edges of the tetrahedron labeled and given a conventional sense indicated by arrows.

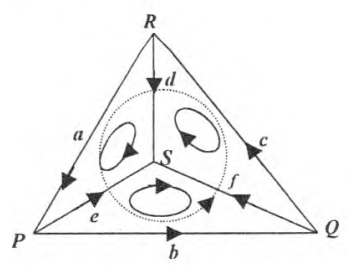

Figure 4 
The vectors representing the faces of the tetrahedron expressed in terms of the edges are: Triangle RSP (note order of vertices coinciding with sense of traversal of ellipse): $1 / 2 \boldsymbol{d} \times(-\boldsymbol{e})$; triangle QSR: $1 / 2$ $\boldsymbol{f} \times(\boldsymbol{( d )}$; triangle PSQ: $1 / 2 \boldsymbol{e} \times(-\boldsymbol{f})$; triangle PQR: $1 / 2$ $\boldsymbol{b} \times \boldsymbol{c}$. Adding the four vectors, noting that since $\boldsymbol{b}=\boldsymbol{e}-\boldsymbol{f}$ and $\boldsymbol{c}=\boldsymbol{f}-\boldsymbol{d}$, we can write the fourth vector product $1 / 2(\boldsymbol{e}-\boldsymbol{f}) \times(\boldsymbol{f}-\boldsymbol{d})=1 / 2[\boldsymbol{e} \times \boldsymbol{f}-\boldsymbol{e} \times \boldsymbol{d}-\boldsymbol{f} \times \boldsymbol{f}+\boldsymbol{f} \times \boldsymbol{d}]$ we obtain

$1 / 2|d \times(-e)+f \times(-d)+e \times(-f)+e \times f-e \times d-f \times f+f \times d|=0$

where we have used the distributive law of the cross product respecting the order of the factors and the facts that for any vectors $\boldsymbol{v}, \boldsymbol{w}: \boldsymbol{w} \times \boldsymbol{w}=0, \boldsymbol{v} \times \boldsymbol{w}=-$ $\boldsymbol{w} \times \boldsymbol{v}$, and $\boldsymbol{w} \times(-\boldsymbol{v})=-\boldsymbol{w} \times \boldsymbol{v}$.

After establishing that, having chosen the orientations of the faces of the tetrahedron in an appropriate manner, either all vector representations of the faces entering or all exiting the tetrahedron, the sum of their vector representations which we will call $\boldsymbol{v}_{1}, \boldsymbol{v}_{2}, \boldsymbol{v}_{3}, \boldsymbol{v}_{4}$, is the zero vector, we can write

$$
v_{1}+v_{2}+v_{3}+v_{4}=0
$$

from which

$$
-v_{1}=v_{2}+v_{3}+v_{4}
$$

Taking the dot product of each member with itself one obtains

$$
\begin{gathered}
v_{1} \cdot v_{1}=\left(v_{2}+v_{3}+v_{4}\right) \cdot\left(v_{2}+v_{3}+v_{4}\right)=v_{2} \cdot v_{2}+ \\
v_{3} \cdot v_{3}+v_{4} \cdot v_{4}+2 v_{2} \cdot v_{3}+2 v_{2} \cdot v_{4}+2 v_{3} \cdot v_{4}
\end{gathered}
$$

which can be written

$$
\begin{aligned}
v_{1}{ }^{2}= & v_{2}{ }^{2}+v_{3}{ }^{2}+v_{4}{ }^{2}+2 v_{2} v_{3} \cos \left(\boldsymbol{v}_{2}, \boldsymbol{v}_{3}\right)+2 v_{2} \\
& v_{4} \cos \left(\boldsymbol{v}_{2}, \boldsymbol{v}_{4}\right)+2 v_{3} v_{4} \cos \left(\boldsymbol{v}_{3}, \boldsymbol{v}_{4}\right)
\end{aligned}
$$

where $v_{1}=\left|v_{1}\right|, v_{2}=\left|v_{2}\right|, v_{3}=\left|v_{3}\right|$, and $v_{4}=\left|v_{4}\right|$ and the angles $\left(\boldsymbol{v}_{i}, \boldsymbol{v}_{i}\right)$ are the angles between the vectors $\boldsymbol{v}_{\boldsymbol{i}}$ and $\boldsymbol{v}_{i}$ representing faces $i$ and $j$ of the tetrahedron, which, because they are orthogonal to the planes of the faces and because of the way we oriented the faces are equal to the diedral angles $\left[180-\left(\boldsymbol{v}_{\boldsymbol{i}}, \boldsymbol{v}_{\boldsymbol{i}}\right)\right]$ between faces $i$ and $j$. Thus if we write the last formula in terms of the diedral angles $\left(p_{i}, p_{j}\right)$ between the planes of face $i$ and face $j$ we have the following expression for the extension of the Law of Cosines for the tetrahedron.

$$
\begin{gathered}
v_{1}{ }^{2}=v_{2}{ }^{2}+v_{3}^{2}+v_{4}{ }^{2}-2 v_{2} v_{3} \cos \left(p_{2}, p_{3}\right)-2 v_{2} v_{4} \cos \\
\left(p_{2}, p_{4}\right)-2 v_{3} v_{4} \cos \left(p_{3}, p_{4}\right)
\end{gathered}
$$

If one of the vectors, say $v_{4}$, is zero, then the tetrahedron becomes a triangle and the formula reduces to the Standard Law of Cosines for the Triangle, namely

$$
v_{1}^{2}=v_{2}^{2}+v_{3}^{2}-2 v_{2} v_{3} \cos \left(p_{2}, p_{3}\right)
$$

It is for this reason that we are justified in calling the formula that was deduced the Extended or Generalized Law of Cosines.

\section{Example of Numerical Verification of the Law of Cosines for Tetrahedra}

Consider a tetrahedron whose four vertices have the following coordinates in a Cartesian coordinate system: $A=(1,0,0), B=(0,1,0), C=(0,0,1), D=(0,2$, $0.2,0)$. The tetrahedron is shown in figure 5 .

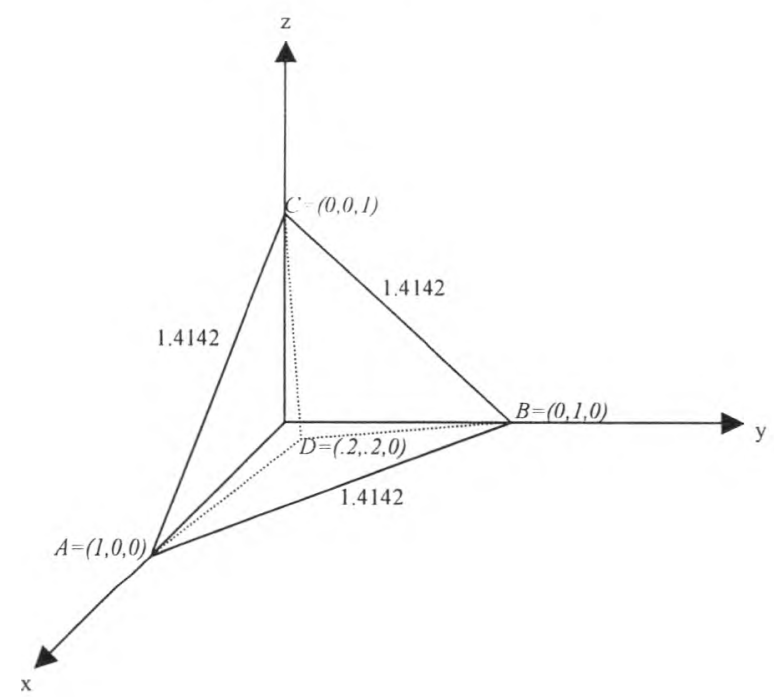

Figure 5

The areas of the triangular faces of the tetrahedron are as follows: $\triangle A B C=1 / 2 \sqrt{3} \triangle A B D=0.3, \triangle A C D=\triangle B C D=$ $\sqrt{0.180}$. Two faces of the tetrahedron are isoceles triangles and their areas can be obtained easily taking one 
half of the product the base and height using the formula $\sqrt{\left(x_{2}-x_{1}\right)^{2}+\left(y_{2}-y_{1}\right)^{2}+\left(z_{2}-z_{1}\right)^{2}}$ to calculate the distance between convenient points $\left(x_{i}, y_{i}, z_{i}\right), i=1$, 2 whose coordinates are known. For the triangles $\triangle A C D$ and $\triangle B C D$ we can use Heron's formula (Thomas et al., 1960)

$$
A=\sqrt{s(s-a)(s-b)(s-c)} \text {, where } s=1 / 2(a+b+c),
$$

and $a, b, c$ are the lengths of the sides of the triangle. Because of symmetry, the areas of triangles $\triangle A C D$ and $\triangle B C D$ are equal, so only one has to be calculated.

The next thing needed to apply the Law of Cosines is to find the diedral angles between adjacent planes which are the suplements to the angles between the normals to the planes. To find a vector normal to a plane determined by three points a simple method is to find the cross product of two vectors lying on the plane. One half of this product will represent the plane area of each triangular face. The vectors can be obtained as the difference of position vectors of pairs of vertices. For the following triangles with the vertices ordered in such a way that the vectors representing the areas enter the tetrahedron, such pairs of vectors are: $\triangle \mathrm{ADC}$ : $(-.8, .2,0),(-.2,-.2,1) ; \triangle \mathrm{BDA}:(.2,-.8,0),(.8,-.2,0)$; $\triangle \mathrm{BCD}:(0,-1,1),(.2, .2,-1) ; \triangle \mathrm{ACB}:(-1,0,1),(0,1$, $-1)$. The corresponding vector areas are: $\triangle A D C$ : $(0.05,0.2,0.05) ; \triangle B D A:(0,0,0.15) ; \triangle B C D:(0.2$, $0.05,0.05) ; \triangle A C B:(-0.25,-0.25,-0.25)$.

The vector sums of the vectors representing the faces of the tetrahedron is the zero vector as expected. The magnitudes of the vectors should be equal to the areas of the triangular faces. We can independently calculate these magnitudes using the formula

$$
|w|=\sqrt{w_{1}^{2}+w_{2}^{2}+w_{3}^{2}}
$$

obtaining Area $\triangle \mathrm{ADC}=\sqrt{0.18}, \triangle \mathrm{BDA}=0.3, \triangle \mathrm{BCD}=$ $\sqrt{0.18}, \triangle \mathrm{ACB}=\sqrt{3} / 2$. The values coincide with those calculated through other means. Finally, if we take the area of one of the faces of the tetrahedron, say $\triangle A C B$, then the square of this area is related to the squares of the areas of the other faces by the Extended Law of Cosines $a^{2}=b^{2}+c^{2}+d^{2}-2 b c \cos (b, c)-2 b d \cos (b, d)-2$ $c d \cos (c, d)$

In this extended law the cosines of the diedral angles between the faces appearing on the right side of the equation need not be calculated explicitly, the terms containing them can be more easily calculated with dot products of the vectors representing the areas because

$$
v_{1} \cdot v_{2}=\left|v_{1}\right|\left|v_{2}\right| \cos \left(v_{1}, v_{2}\right)
$$

The Law of Cosines then becomes

$$
\begin{gathered}
(\triangle \mathrm{ACB})^{2}=(\triangle \mathrm{ADC})^{2}+(\triangle \mathrm{BCD})^{2}+(\triangle \mathrm{BDA})^{2}+ \\
2 \mathrm{ADC}+\mathrm{BC} \mathrm{C}+2 \mathrm{ADC} \cdot \overrightarrow{\mathrm{BDA}}+2 \mathrm{BC} \cdot \overrightarrow{\mathrm{BDA}}
\end{gathered}
$$

In this formula plus signs appear because the angles to be used in the dot products are the angles between the vectors representing the areas which are suplementary to the diedral angles between the faces which appear in the previous formula. When we introduce the numerical values in the last formula we obtain

$$
\begin{gathered}
0.75=0.18+0.18+0.18+0.09+0.18+0.06 \\
+0.06
\end{gathered}
$$

Since this last equation is correct, we have an instance of a numerical verification of the Extended Law of Cosines.

\section{A Determinantal Expression for the Area of a Triangle}

Consider figure 6, where lines $\mathrm{P}_{3} \mathrm{P}_{x 3}, \mathrm{P}_{2} \mathrm{P}_{x 2}, \mathrm{P}_{1} \mathrm{P}_{x 1}$ are orthogonal to the $x$ axis

$\mathrm{P}_{2}$

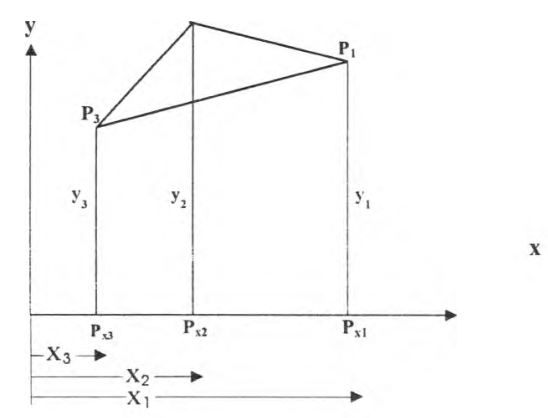

Figura 6 
Area $\Delta \mathrm{P}_{1} \mathrm{P}_{2} \mathrm{P}_{3}=$ Area Trapezoid $\mathrm{P}_{2} \mathrm{P}_{3} \mathrm{P}_{x 3} \mathrm{P}_{x 2}+$ Area Trapezoid $\mathrm{P}_{1} \mathrm{P}_{2} \mathrm{P}_{x 2} \mathrm{P}_{x 1}-$ Area Trapezoid $\mathrm{P}_{1} \mathrm{P}_{3} \mathrm{P}_{x 3} \mathrm{P}_{x 1}$

The area of each trapezoid is equal to one half of the sum of the bases times the distance between them, therefore

$$
\begin{aligned}
& \text { Area } \Delta \mathrm{P}_{1} \mathrm{P}_{2} \mathrm{P}_{3}=1 / 2\left(y_{2}+y_{3}\right)\left(x_{2}-x_{3}\right)+1 / 2\left(y_{2}+y_{1}\right)\left(x_{1}\right. \\
& \left.\quad-x_{2}\right)-1 / 2\left(y_{1}+y_{3}\right)\left(x_{1}-x_{3}\right) \\
& =1 / 2\left|x_{2} y_{2}+x_{2} y_{3}-x_{3} y_{2}-x_{3} y_{3}\right|+1 / 2 \mid x_{1} y_{2}+x_{1} \\
& y_{1}-x_{2} y_{2}-x_{2} y_{1}|-1 / 2| x_{1} y_{1}+x_{1} y_{3}-x_{3} y_{1}-x_{3} y_{3} \mid
\end{aligned}
$$

which, after cancelling terms with opposite signs, becomes

$$
\text { Area } \begin{gathered}
\Delta \mathrm{P}_{1} \mathrm{P}_{2} \mathrm{P}_{3}= \\
1 / 2\left|x_{2} y_{3}+x_{1} y_{2}+x_{3} y_{1}\right|-1 / 2 \mid x_{3} y_{2} \\
+x_{2} y_{1}+x_{1} y_{3} \mid
\end{gathered}
$$

The right hand side can be writen as a determinant, so we finally have

$$
\text { Area } \Delta \mathrm{P}_{1} \mathrm{P}_{2} \mathrm{P}_{3}=1 / 2\left|\begin{array}{lll}
x_{1} & y_{1} & 1 \\
x_{2} & y_{2} & 1 \\
\chi_{3} & y_{3} & 1
\end{array}\right|
$$

The reader can verify that for arbitrary values of the coordinates of the three points $\mathrm{P}_{1}, \mathrm{P}_{2}, \mathrm{P}_{3}$, the final expression for the area of the triangle is the same. From the numerical point of view, if the points are ordered in such a way that when they are traversed in natural order the perimeter is traversed in the counterclockwise sense the area will be a positive number, for the given orientation of the axes, while if the sense of the traversal is clockwise the area will be negative. This circumstance is compatible with the property of determinants that if any two rows of the determinant are interchanged the determinant changes sign. The rule is also compatible with the convention of the right hand screw. Similar expressions can be derived for the volumes of tetrahedrons and hypervolumes of simplices of higher dimensions. (See paragraph Generalization of the Law of Cosines for Four and More Dimensions for a definition of an $n$-dimensional simplex).

For an $n$-dimensional simplex the expression for its hypervolume $V_{n}$ is

$$
V_{n}=\frac{1}{n !}\left|\begin{array}{ccccc}
\chi_{1}^{1} & \chi^{2}{ }_{1} & \ldots & \chi^{{ }_{1}}{ }_{1} & 1 \\
\chi^{1}{ }_{2} & \chi^{2}{ }_{2} & \ldots & \chi^{n_{2}} & 1 \\
\ldots & \ldots & \ldots & \ldots & \ldots \\
\chi_{n+1}^{1} & \chi^{2}{ }_{n+1} & \ldots & \chi^{n}{ }_{n+1} & 1
\end{array}\right|
$$

Where the subindices denote points and the superindices refer to the labels on the axes (they are not exponents).

\section{Alternative Proof of the Law of Cosines for the Tetrahedron}

Consider the following determinants

$$
\left|\begin{array}{ll}
x_{1} & 1 \\
x_{2} & 1
\end{array}\right|, 1 / 2\left|\begin{array}{lll}
x_{1} & y_{1} & 1 \\
x_{2} & y_{2} & 1 \\
x_{3} & y_{3} & 1
\end{array}\right|, 1 / 6\left|\begin{array}{llll}
x_{1} & y_{1} & z_{1} & 1 \\
x_{2} & y_{2} & z_{2} & 1 \\
x_{3} & y_{3} & z_{3} & 1 \\
x_{4} & y_{4} & z_{4} & 1
\end{array}\right|
$$

The first one gives us an expression for the signed magnitude (length) of a line (one dimensional figure) whose bordering elements are two points which in a one-dimensional space with a single Cartesian axis coincident with the line containing the two points have coordinates $x_{1}$ and $x_{2}$. In figure 7 a) we show the axis oriented in such a way that the length is positive. An opposite orientation either of the axis or of the order of the points would result in a change of sign of the length.

The second determinant gives us an expression for the signed magnitude (area) of a triangle (two dimensional figure) whose bordering elements are three oriented lines lying in two-dimensions with two cartesian axes lying on the plane containing the three points which have pairs of coordinates $\left(x_{1}, y_{1}\right),\left(x_{2}, y_{2}\right)$, $\left(x_{3}, y_{3}\right)$. In figure $7 \mathrm{~b}$ ) we show the axes oriented in such a way that the area is positive. An odd permutation of the points or reversal of an odd number of axes would result in a change of sign of the area.

The third determinant gives us an expression for the signed magnitude (volume) of a tetrahedron (three-dimensional figure) whose bordering elements are four oriented triangles lying in three dimensions with three Cartesian axes lying in the three dimensional space containing the four points which have trios of coordinates $\left(x_{1}, y_{1}, z_{1}\right),\left(x_{2}, y_{2}, z_{2}\right),\left(x_{3}, y_{3}, z_{3}\right),\left(x_{4}, y_{4}, z_{4}\right)$. In figure $7 \mathrm{c}$ ) we show the axes oriented in such a way that the volume is positive. An opposite orientation of an 
odd number of axes or an odd permutation of the points would result in a change of sign of the volume.

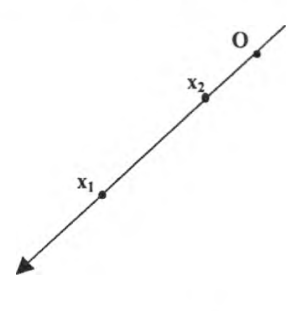

a)

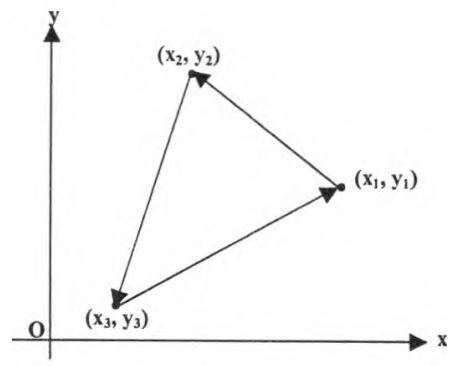

b)

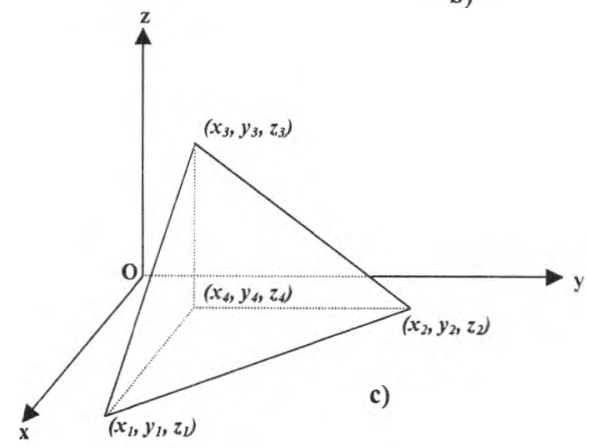

Figure 7
In figure 7 the "bodies" are placed in an arbitrary position. If, however, we made the origin coincide with the highest numbered vertex in each figure, the coordinates would be transformed with each new coordinate equal to the old coordinate minus the corresponding coordinate of the highest numbered vertex. The determinants would not change values because of the property of a determinant which allows the addition to any line of a multiple of another line multiplied by a constant. We illustrate the operation for the case of the triangle (corresponding to figure $7 \mathrm{~b}$ ) and the second determinant, ignoring for the moment the multiplier $1 / 2$ ).

$$
\left|\begin{array}{ccc}
x_{1}-x_{3} & y_{1}-y_{3} & 0 \\
x_{2}-x_{3} & y_{2}-y_{3} & 0 \\
x_{3} & y_{3} & 1
\end{array}\right|=\left|\begin{array}{cc}
x_{1}-x_{3} & y_{1}-y_{3} \\
x_{2}-x_{3} & y_{2}-y_{3}
\end{array}\right|=\left|\begin{array}{cc}
x_{1}^{\prime} & y_{1}^{\prime} \\
x_{2}^{\prime} & y_{2}^{\prime}
\end{array}\right|
$$

Starting with the second determinant of $\left({ }^{*}\right)$ we add to the second row the third row multiplied by -1 and also add to the first row the third row multiplied by -1 . We then expand the resulting determinant by cofactors (signed minors) of the elements of the third column. By calling the coordinates $x_{1}{ }^{\prime}=x_{1}$ $x_{3}, y_{1}{ }^{\prime}=y_{1}-y_{3}, x_{2}{ }^{\prime}=x_{2}-x_{3}, y_{2}{ }^{\prime}=y_{2}-y_{3}$, we get the final determinant. That the value of the determinant multiplied by $1 / 2$ is the area of the triangle was determined in the analysis associated with figure 6 . Similar developments show that the third determinant of $\left({ }^{*}\right)$ is equal to the volume (ignoring the sign) of a parallelepided whose three edges meeting in a vertex are the vectors whose components are $\left(x_{1}{ }^{\prime}\right.$, $\left.y_{1}{ }^{\prime}, z_{1}{ }^{\prime}\right),\left(x_{2}{ }^{\prime}, y_{2}{ }^{\prime}, z_{2}{ }^{\prime}\right),\left(x_{3}{ }^{\prime}, y_{3}{ }^{\prime}, z_{3}{ }^{\prime}\right)$, where $x_{i}{ }^{\prime}=x_{i}-x_{4}$, $y_{i}^{\prime}=y_{i}-y_{4}, z_{i}{ }^{\prime}=z_{i}-z_{4} ; i=1,2,3$. The factor $1 / 6$ for the tetrahedron comes from the fact that the triangles, instead of parallelograms, contribute $1 / 2$ and the pyramids (a tetrahedron is a triangular pyramid) instead of parallelepipeds, which are prisms, contribute the factor $1 / 3$. (Thomas, 1960; Keedy y Nelson, 1965 ).

We now recall the property of a determinant that says that if in the expansion of a determinant by the elements and cofactors of a given column the elements of the column are replaced by the elements of a different column the result is zero. The reason for this comes from the fact that in replacing the elements of the column in terms of which the expansion is made by the elements of a different column, what we are doing is expanding the determinant of an array that has two identical columns, therefore the value of that determinant is zero. (Hildebrand et al., 1952)

Ignoring for the moment the factor $1 / 6$, let us expand the third determinant of $(*)$ expressing the volume of a tetrahedron in terms of the cofactors of the first column but replacing the elements of the first column by the elements of the fourth column; the result should be zero

$$
\begin{gathered}
\left|\begin{array}{llll}
1 & y_{1} & z_{1} & 1 \\
1 & y_{2} & z_{2} & 1 \\
1 & y_{3} & z_{3} & 1 \\
1 & y_{4} & z_{4} & 1
\end{array}\right|=\left|\begin{array}{lll}
y_{2} & z_{2} & 1 \\
y_{3} & z_{3} & 1 \\
y_{3} & z_{4} & 1
\end{array}\right|-\left|\begin{array}{lll}
y_{1} & z_{1} & 1 \\
y_{3} & z_{3} & 1 \\
y_{4} & z_{4} & 1
\end{array}\right|+ \\
\left|\begin{array}{lll}
y_{1} & z_{1} & 1 \\
y_{2} & z_{2} & 1 \\
y_{4} & z_{4} & 1
\end{array}\right|-\left|\begin{array}{lll}
y_{1} & z_{1} & 1 \\
y_{2} & z_{2} & 1 \\
y_{3} & z_{3} & 1
\end{array}\right|=0
\end{gathered}
$$

Let us see what is the meaning of the four third order determinants in the previous equation. According to the analysis associated with figure 6 they correspond to the areas of four triangles 
having as vertices the same points as the vertices of the tetrahedron except that in each determinant one of the points is missing and in all four determinants the $x$ component is missing. A moment's reflection reveals that what we have is the projection upon the $y-z$ plane of each of the areas of the faces of the tetrahedron. The equation therefore expresses that the algebraic sum of the projections of the signed areas of the faces of the tetrahedron upon the $y-z$ plane is zero, which is the same as saying that the vector sum of the projections of the vectors representing the areas of the faces upon a unit vector normal to the $y-z$ plane (the unit vector on the $x$ axis) is zero.

We now repeat the process but now expanding the determinant expressing the volume of the tetrahedron with respect to the cofactors of the second column replacing the values of the elements of the second column by the elements in the fourth column, and again obtain a zero sum. We observe that in this expansion no $y$ components appear. This new expansion would represent the algebraic sum of the projections of the signed areas of the faces of the tetrahedron upon the $x-z$ plane, which would be equal to the projections of the vectors representing said faces upon a unit vector normal to the $x-z$ plane (the unit vector on the $y$ axis.) The result is zero.

Finally we repeat the process but now expanding the determinant expressing the volume of the tetrahedron with respec to the cofactors of the third column replacing the elements of the third column by the elements of the fourth column, and again obtain a zero sum. We observe that in this expansion no $z$ components appear. This expansion would indicate that the algebraic sum of the projections of the signed areas of the faces of the tetrahedron upon the $x-y$ plane is zero, which would be the same as saying that the vector sum of the projections of the vectors representing the areas of the faces upon a unit normal vector to the $x-y$ plane (the unit vector on the $z$ axis) is zero.

But having proved that the three projections with respect to an orthonormal basis of a vector are zero, we have to conclude that the vector is the zero vector. Having established that the vector sum of the vectors representing the the signed areas of the faces of a tetrahedron is zero, the Law of Cosines follows using the procedure consisting in writing in the left side of an equation the negative of one of the summands and the sum of the other vectors and obtaining through the dot product the lengths of both members of the equations as done in the beginning of this article. Although we had proven this using the cross product, the merit of this alternative proof is that it is easily generalized to more than three dimensions as explained in the following section.

\section{Generalization of the Law of Cosines for Four and More Dimensions}

In going from three to four and more dimensions in which there is no direct physical geometric interpretation, we have to rely on analogy, definitions, and algebra (Courant y Robbins, 1941). We start by defining a simplex.

A simplex in an $n$-dimensional Euclidean space is the convex hull of $n+1$ points not all of which lie in an (n-1) dimensional manifold. (Dubrovin et al., 1987)

A simplex can be constructed inductively as follows: A one dimensional simplex is the line segment between two points. A two dimensional simplex is the set of points in a triangle. If we have an $n$-dimensional simplex in an $n$-dimensional space $\Re^{n}$ then if a new vertex is taken outside of this space in an $(n+1)$-dimensional space of which the original $n$-dimensional space is a subset (a hyperplane), the union of the points on the line segments joining the added vertex with each of the points of the original $n$-dimensional simplez is an $(n+1)$-dimensional simplex.

There are several concepts in these definitions that require clarification. A set of points is convex if whenever any two points belong to the set all the points lying in the segment of line connecting them also belong to the set. (Hillier y Lieberman, 1974) The convex hull of a finite set of points is the smallest convex set containing the original points. The convex hull of a finite set of points can also be defined as the set of points that can be obtained as a convex linear combination of the original points, (Wagner, 1969). That is, if $x_{1}, x_{2}, \ldots, x_{n}$ are the original points, a convex linear combination of these 
points is a point $x$ that satisfies $x=r_{1} x_{1}+r_{2} x_{2}+\ldots$ $+r_{n} x_{n}$, where the real numbers $r_{1}, r_{2}, \ldots, r_{n} \geq 0$ and $r_{1}+r_{2}+\ldots+r_{n}=1$. Informally speaking, in a plane the convex hull of a finite set of points is the polygon formed by a rubber band that starting from a circle large enough to contain all the points is released and takes as vertices of the polygon the "outer" points, if we imagine that at each point we place a nail and the nails limit the shrinking of the rubber band. See figure 8 .

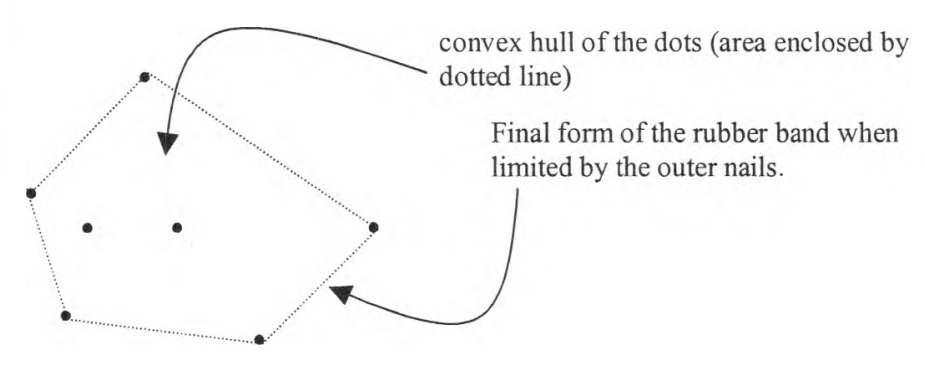

Figure 8

In a three dimensional space the convex hull of a finite set of points is a convex polyhedron.

A Euclidean space is ordinary space as we sense it, in which the shortest distance between two points is that given by a straight line. We can restrict our attention to one or two dimensions and speak of the latter as the space of a plane. (For instance, plane geometry studies figures in a plane.) It is also a space that is uniform in all directions, the same unit of distance applies. When we extend the concepts to $n$ dimensions we have to formalize. We introduce orthonormal (orthogonal and of unit length) Cartesian axes and coordinates related to them The principal concepts are the concept of distance which is defined via the inner product between vectors and the concept of angle, also defined via the inner product.

The inner product of two vectors $x$ and $y$ in a Euclidean space of $n$ dimensions can be defined as

$$
\mathrm{x} \bullet \mathrm{y}=\mathrm{x}_{1} y_{1}+x_{2} y_{2}+\ldots+x_{n} y_{n}
$$

The length $|x|$ of a vector $x$ can then be defined as

$$
|x|=\sqrt{x_{1}^{2}+x_{2}{ }^{2}+\ldots+x_{n}{ }^{2}}=\sqrt{x \cdot x}
$$

and the angle $\theta$ between two vectors $v, w$ through its cosine

$$
\cos \theta=(u \bullet v) /|u||v|
$$

The signed "volume" of an $n$-dimensional parallelepiped whose edges meet in a vertex and are represented by the vectors $v_{1}, v_{2}, \ldots, v_{n}$ (we will call this set of vectors $\boldsymbol{v}$ ) is defined as follows (Xambó, 1997)

$$
V(v)=\operatorname{det} \mathbf{M}
$$

Where $\mathbf{M}$ is the matrix whose columns are the representations of the vectors $v_{1}, v_{2}, \ldots, v_{n}$ with respect to any orthonormal basis, that is, its i-th column is formed with the coefficients of the expansion of $v_{i}$ as a linear combination of the orthonormal basis vectors. This definition of the signed volume is a straightforward extension of the formula of the signed volume of a line segment, a parallelogram and a three-dimensional parallelepiped. To calculate the volume of a simplex which is the extension of a line segment, a triangle and a tetrahedron all we have to add is a factor $(n !)^{-1}$ where $n$ is the number of dimensions. Thus for the segment the factor is 1 ! $=1$, for a triangle the factor is $1 / 2 !=1 / 2$, for the tetrahedron the factor is $1 / 3 !=1 / 6$. For an $n$-dimensional simplex the factor is $1 / n !$.

In order to intuitively understand the reason for this factor in figure 9 we show for the two-dimensional parallelogram and for the three-dimensional parallelepiped the subdivision of its "volume" in $n$ ! simplices of equal "volume."

Now we proceed with the proof of the Law of Cosines for $n$-dimensional simplices. (By speaking of $n$ ! times the volume, in the following we supress the $n$ ! factor from the discussion.) Proceeding as before we can express schematically

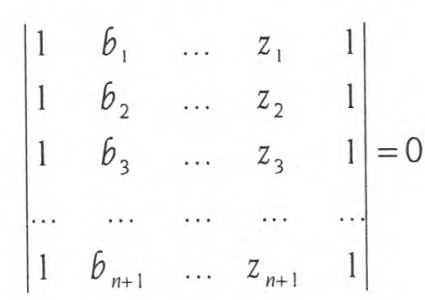

expression with $n$ terms with alternating signs, each term with an n-order minor of one of the units in the leftmost column, in which $a$ (the first of the $n$ variables $a, b \ldots \ldots$ does not appear, while all the others are present). In each term one of the subindices does not appear indicating it corresponds to a "face" in which one of the vertices is missing. The expression represents the projections of all $n+1(n-1)-$ dimensional simplices ("faces") that border (or form the "skin" of) the n-dimensional simplex. 
This scheme is repeated by placing the column of ones in the second, third, ..., n-th column and expanding the determinant via the cofactors of said column. The resulting expressions indicate that the vectors representing the "faces" of the simplex have projections on all the axes that algebraically add to zero in each instance.

For clarity only one half of the parallelepiped is divided into three tetrahedra (simplices in three dimensions.) Each of the three tetrahedra have the same volume because the tetrahedra $\mathrm{P}_{1} \mathrm{P}_{2} \mathrm{P}_{3} \mathrm{P}_{5}$ and $\mathrm{P}_{3} \mathrm{P}_{2} \mathrm{P}_{6} \mathrm{P}_{5}$ have equal bases (one half of the area of parallelogram $\mathrm{P}_{1} \mathrm{P}_{2} \mathrm{P}_{6} \mathrm{P}_{3}$ ) and the same height (the distance between parallelograms $\mathrm{P}_{1} \mathrm{P}_{3} \mathrm{P}_{6} \mathrm{P}_{2}$ and point $\mathrm{P}_{5}$.) On the other hand tetrahedra $\mathrm{P}_{1} \mathrm{P}_{2} \mathrm{P}_{3} \mathrm{P}_{5}$ and $\mathrm{P}_{3} \mathrm{P}_{4} \mathrm{P}_{6} \mathrm{P}_{5}$ have equal volume because they have as bases one half of equal parallelograms on the two horizontal planes and have equal heights (distance between horizontal parallelograms. By transitivity the three tetrahedra have equal volume. Hence by subdividing both halves of the parallelepiped, it can be divided into 6 tetrahedra of equal volume. The argument can be extended to simplices of more dimensions by cutting an $n$ dimensional parallelepiped into $n$ ! equal $n$-dimensional simplices. (Xambo-Descamps 1997).

Figure 9

The conclusion is that the polygon of vectors representing the faces of the simplex that correspond to their sum have a zero projection on $n$ linearly independent vectors in an $n$-dimensional space, a fact which implies that the sum of the vectors is the zero vector. From that fact the Law of Cosines easily follows using the same scheme as for the triangle and the tetrahedron.

\section{Numerical Verification of the Law of Cosines for a Four-Dimensional Simplex}

The method of proof using determinants allowed us to easily extend the ideas used for the tetrahedron to simplices in four and more dimensions. The proof is completely analogous. To clarify the process avoiding complicated notation we will provide a numerical example for a four-dimensional simplex. If we refer to a Cartesian coordinate system in four dimensions with axes $w, x, y, z, a$ simplex having vertices $\left(w_{1}, x_{1}, y_{1}, z_{1}\right),\left(w_{2}, x_{2}, y_{2}, z_{2}\right)$, $\left(w_{3}, x_{3}, y_{3}, z_{3}\right),\left(w_{4}, x_{4}, y_{4}, z_{4}\right)$, will by definition have twenty four times its volume given by a fourth order determinant in terms of four vectors whose components are obtained from the coordinates of the vertices diminished by the coordinates of the vertex which is chosen to coincide with the origin. By using the property of determinants that allow us to replace a row by the result of adding to the row another row multiplied by a constant without changing the value of the determinant, we can write the following equality among determinants

$$
24 \mathrm{~V}=\left|\begin{array}{llll}
w_{1}-w_{5} & x_{1}-x_{5} & y_{1}-y_{5} & z_{1}-z_{5} \\
w_{2}-w_{5} & x_{2}-x_{5} & y_{2}-y_{5} & z_{2}-z_{5} \\
w_{3}-w_{5} & x_{3}-x_{5} & y_{3}-y_{5} & z_{3}-z_{5} \\
w_{4}-w_{5} & x_{4}-x_{5} & y_{4}-y_{5} & z_{4}-z_{5}
\end{array}\right|=\left|\begin{array}{lllll}
w_{1} & x_{1} & y_{1} & z_{1} & 1 \\
w_{2} & x_{2} & y_{2} & z_{2} & 1 \\
w_{3} & x_{3} & y_{3} & z_{3} & 1 \\
w_{4} & x_{4} & y_{4} & z_{4} & 1 \\
w_{5} & x_{5} & y_{5} & z_{5} & 1
\end{array}\right|
$$

What we have done to establish the equality is to take the determinant on the right hand side and replace rows one to four by the same rows minus the fifth row to end with a fifth order determinant equal to the fourth-order determinant on the left side 
bordered at the bottom with the fifth row of the determinant on the right and a column on the right having zeros everywhere except in the fifth place wich has unity there. A subsequent expansion of the fifth order determinant in terms of the fifth column gives the determinant on the left. Let us consider now a four-dimensional simplex defined by the following vertices: $P_{1}=(1,0,0,0), P_{2}=(0,1,0,0), P_{3}$ $=(0,0,1,0), P_{4}=(0,0,0,1), P_{5}=(0.2,0.2,0,0) . A$ three-dimensional projection of the simplex projected unto two dimensions is shown in figure 10.

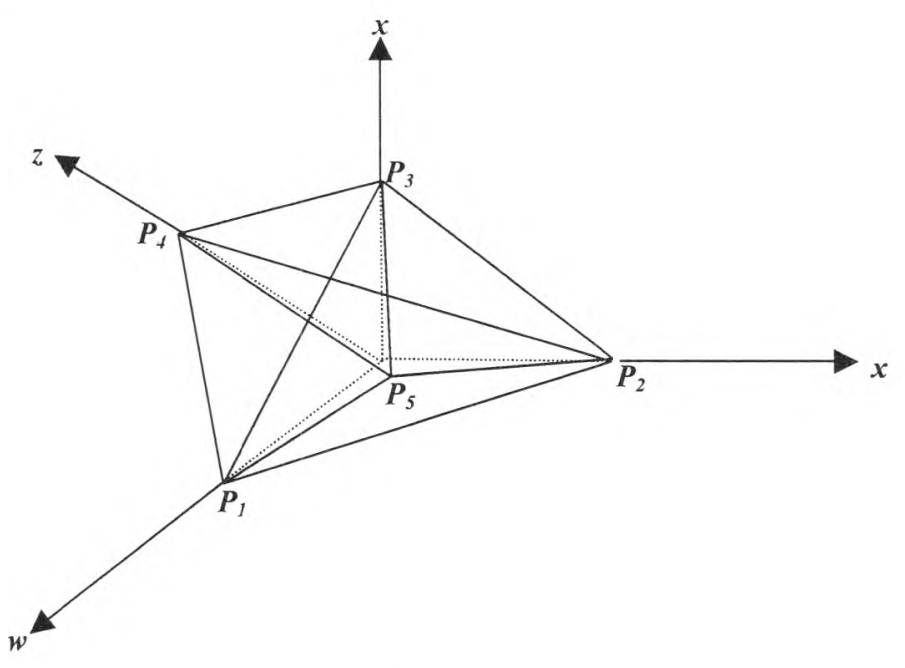

Figure 10

(This simplex is obtained from the first tetrahedron used above to verify the Law of Cosines for tetrahedra by adding a fifth point - and placing it in fourth place - placed a unit of distance away from the origin on a fourth axis simultaneously orthogonal to the first three. The coordinates of this additional vertex are $(0,0,0,1)$.

The expansion of $6 \mathrm{~V}$ is shown below with dotted lines showing columns and rows which disappear on expanding by columns intermediate determinants. The third order determinant is evaluated directly. The final result is the magnitude of $6 \mathrm{~V}$ of the four-dimensional simplex in units of length to the fourth power.

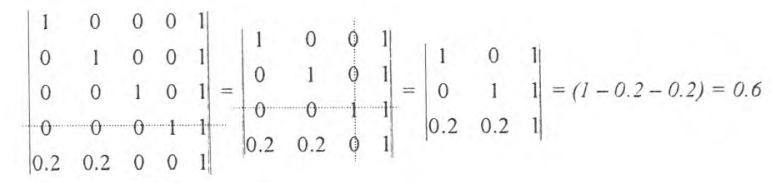

By using the idea of replacing the first column with the fifth column in the determinant on the right hand side and expanding the resulting determinant which we know is zero, we can get an expression involving a linear combination of determinants which can be interpreted as the projections on the axis $w$ of vector representations of the volumes of the pieces of hyperplanes which enclose the simplex. In the case of the four dimensional simplex these pieces of hiperplanes are tetrahedrons which we will call $T_{1}, T_{2}, T_{3}, T_{4}$ and $T_{5}$, and their projection on the $w$ axis will be called $T_{1}{ }^{w}, T_{2}{ }^{w}, T_{3}{ }^{w}, T_{4}{ }^{w}, T_{5}{ }^{w}$.

$$
\begin{aligned}
\left|\begin{array}{lllll}
1 & x_{1} & y_{1} & z_{1} & 1 \\
1 & x_{2} & y_{2} & z_{2} & 1 \\
1 & x_{3} & y_{3} & z_{3} & 1 \\
1 & x_{4} & y_{4} & z_{4} & 1 \\
1 & x_{5} & y_{5} & z_{5} & 1
\end{array}\right| & =\left|\begin{array}{llll}
x_{2}{ }^{\prime \prime} & y_{2} & z_{2} & 1 \\
x_{3} & y_{3} & z_{3} & 1 \\
x_{4} & y_{4} & z_{4} & 1 \\
x_{5} & y_{5} & z_{5} & 1
\end{array}\right|-\left|\begin{array}{llll}
T_{2}{ }^{\prime \prime} & \\
x_{1} & y_{1} & z_{1} & 1 \\
x_{3} & y_{3} & z_{3} & 1 \\
x_{4} & y_{4} & z_{4} & 1 \\
x_{5} & y_{5} & z_{5} & 1
\end{array}\right|+\left|\begin{array}{llll}
x_{1} & T_{3}{ }^{\prime \prime} & z_{1} & 1 \\
x_{2} & y_{2} & z_{2} & 1 \\
x_{4} & y_{4} & z_{4} & 1 \\
x_{5} & y_{5} & z_{5} & 1
\end{array}\right| \\
& -\left|\begin{array}{llll}
x_{1} & y_{1}{ }^{\prime \prime} & z_{1} & 1 \\
x_{2} & y_{2} & z_{2} & 1 \\
x_{3} & y_{3} & z_{3} & 1 \\
x_{5} & y_{5} & z_{5} & 1
\end{array}\right|+\left|\begin{array}{llll}
x_{1} & y_{1} & z_{1} & 1 \\
x_{2} & y_{2} & z_{2} & 1 \\
x_{3} & y_{3} & z_{3} & 1 \\
x_{4} & y_{4} & z_{4} & 1
\end{array}\right|
\end{aligned}
$$

Each determinant marked with $\mathrm{T}_{i}{ }^{*}, i=1,2,3,4,5$ corresponds to six times the projection on the $w$ axis of the vector representing the volume of the corresponding tetrahedron. The tetrahedrons are the ones defined by all 5 vertices minus the one appearing in its subindex. Thus $T_{1}$ has as vertices $P_{2}, P_{3}, P_{4}, P_{5} ; T_{3}$ has vertices $P_{1}, P_{2}, P_{4}$, and $P_{5}$, etc. After putting in the values of the coordinates of the vertices we can evaluate each determinant. We show the details for the case of $T_{1}$.

$$
T_{1}{ }^{w}=\left|\begin{array}{cccc}
1 & 0 & 0 & 1 \\
0 & 1 & 0 & 1 \\
0 & 0 & 1 & 1 \\
0.2 & 0 & 0 & 1
\end{array}\right|=\left|\begin{array}{ccc}
1 & 0 & 1 \\
0 & 1 & 1 \\
0.2 & 0 & 1
\end{array}\right|=\left|\begin{array}{cc}
1 & 1 \\
0.2 & 1
\end{array}\right|=(1-0.2)=0.8
$$

Doing the same for the other T's we obtain

$$
\mathrm{T}_{2}{ }^{w}=0.2, \mathrm{~T}_{3}{ }^{w}=0, \mathrm{~T}_{4}{ }^{w}=0, \mathrm{~T}_{5}{ }^{w}=-1
$$

Repeating the process but succesively replacing the second, third, and fourth column with ones, and expanding the resulting determinants in terms of these columns, we can calculate the projections 
upon the $x, y, z$ axes of the vectors representing the volumes of the tetrahedra. The results are

$$
\begin{gathered}
\mathrm{T}_{1}{ }^{x}=0.2, \mathrm{~T}_{2}{ }^{x}=0.8, \mathrm{~T}_{3}{ }^{x}=0, \mathrm{~T}_{4}{ }^{x}=0, \mathrm{~T}_{5}^{x}=-1 \\
\mathrm{~T}_{1}{ }^{y}=0.2, \mathrm{~T}_{2}{ }^{y}=0.2, \mathrm{~T}_{3}{ }^{y}=0.6, \mathrm{~T}_{4}^{y}=0, \mathrm{~T}_{5}{ }^{y}=-1 \\
\mathrm{~T}_{1}{ }^{z}=0.2, \mathrm{~T}_{2}^{z}=0.2, \mathrm{~T}_{3}{ }^{z}=0, \mathrm{~T}_{4}^{z}=0.6, \mathrm{~T}_{5}^{z}=-1
\end{gathered}
$$

All these quantities should be multiplied by $1 / 6$ in order to get the actual values since in the whole process we have left out the factor $1 / 6$ in front of the original determinants. We thus have the vector representations of the tetrahedra that enclose the four-dimensional simplex as follows

$$
\begin{aligned}
& \mathrm{T}_{1}=1 / 6(0.8,0.2,0.2,0.2) \\
& \mathrm{T}_{2}=1 / 6(0.2,0.8,0.2,0.2) \\
& \mathrm{T}_{3}=1 / 6(0,0,0.6,0) \\
& \mathrm{T}_{4}=1 / 6(0,0,0,0.6) \\
& \mathrm{T}_{5}=1 / 6(-1,-1,-1,-1)
\end{aligned}
$$

The volumes of the tetrahedrons are the magnitudes of these vectors. Thus we have

$$
\begin{aligned}
& V_{1}=\sqrt{76} / 6 \\
& V_{2}=\sqrt{76} / 6 \\
& V_{3}=0.1 \\
& V_{4}=0.1 \\
& V_{5}=1 / 3
\end{aligned}
$$

A partial check can be obtained by calculating the volumes of the tetrahedra through an independent geometrical process. Let us start with $V_{4}$. The tetrahedron whose volume is $V_{4}$ has vertices $P_{1}$, $P_{2}, P_{3}, P_{5}$. This tetrahedron has been previously analyzed in this paper, although its volume has not been valculated. To calculate its volume we can take as the base of a triangular pyramid the triangle $\Delta \mathrm{P}_{1} \mathrm{P}_{2} \mathrm{P}_{3}$ whose area was previously calculated as 0.3 . The height of the tetrahedron or triangular pyramid is obviously $h=1$, since the base lies in the $\mathrm{w}-\mathrm{x}$ plane. Using the well known formula for the volume of a pyramid

$$
V=(1 / 3) 6 h
$$

Where 6 is the area of the base and $\bar{h}$ the height. Putting the values of $b$ and $h$ in the formula we find

$$
V_{4}=0.3 \times 1 / 3=0.1
$$

Which coincides with the value calculated through the determinants. By inspection of figure 10 , we can see that $V_{3}$ and $V_{4}$ should have the same value, since the $z$ axis is as orthogonal to the plane $w-x$ as is the $y$ axis and the points $P_{3}$ and $P_{4}$ are similarly placed on these axes.

Let us now check $V_{5}$ which corresponds to the tetrahedron with vertices $\mathrm{P}_{1}, \mathrm{P}_{2}, \mathrm{P}_{3}, \mathrm{P}_{4}$. The triangle $\mathrm{P}_{1} \mathrm{P}_{2} \mathrm{P}_{3}$ was also previously analyzed and its area was found to be $1 / 2$ We now have to find the height of the tetrahedron which is the distance of the point $\mathrm{P}_{4}$ to the plane defined by the points $\mathrm{P}_{1}, \mathrm{P}_{2}, \mathrm{P}_{3}$. One way to find this distance is to find a vector that goes from point $\mathrm{P}_{4}=(0,0,0,1)$ to any point in the plane of the triangle, for example point $P_{1}=(1,0,0$, $0)$. The line joining these two points will, in general, not be orthogonal to the plane of the triangle, but it can be orthogonalized by subtracting from the vector its projection on the plane, what is left will have zero projection on the plane, that is, it will be orthogonal to the plane. This can be done by finding two orthogonal vectors in the plane of the triangle and subtracting from the original vector its projections upon the two orthogonal vectors, whatever is left will be orthogonal to the plane because it will have zero projection on the plane. One of the vectors on the plane can be chosen to be one of the edges of the triangle. The other vector should be orthogonal to the chosen edge. If we take the edge going from the vertex $\mathrm{P}_{1}$ to the vertex $\mathrm{P}_{2}$, then an orthogonal vector can be the one going from the midpoint of this edge to vertex $P_{3}$. To easily calculate the projections it is convenient that the two orthogonal vectors on the plane be of unit length. This can be accomplished by dividing the vectors by their lengths.

Let us then implement the strategy described. A vector going from vertex $P_{4}$ to vertex $P_{1}$ is given by the quartet of components

$$
(1,0,0,0)-(0,0,0,1)=(1,0,0,-1)
$$

The vector going from vertex $P_{1}$ to $P_{2}$ is given by the quartet $(0,1,0,0)-(1,0,0,0)=(-1,1,0,0)$ which made unitary becomes $1 / \sqrt{2}(-1,1.0,0)$. 
The vector that goes from the midpoint of the edge $\mathrm{P}_{1} \mathrm{P}_{2}$ to $\mathrm{P}_{3}$ is given by

$$
(0,0,1,0)-(0.5,0.5,0,0)=(-0.5,-0.5,1,0)
$$

which normalized is

$$
(1 / \sqrt{1.5})(-0.5,-0.5,1,0)
$$

The projections of the first vector upon the two orthonormal vectors are

$$
\begin{gathered}
{[(0,0,1,-1) .(-1,1,0,0)(1 / \sqrt{2})](0-1,1,0,} \\
0)(1 / \sqrt{2})=\mathbf{0}
\end{gathered}
$$$$
[(0,0,1,0-1) .(-0.5,-0.5,1,0)(1 / \sqrt{1.5})](-0.5 \text {, }
$$$$
-0.5,1,0)(1 / \sqrt{1.5})=(-1 / 3,-1 / 3,2 / 3,0)
$$

Since the original vector has a non-zero projection upon only one of the unit vectors, the orthogonal vector results from subtracting from the original vector the second projection. We obtain

$$
(0,0,1,-1)-(-1 / 3,-1 / 3,2 / 3,0)=(1 / 3,1 / 3, / 3,-1)
$$

The last vector is orthogonal to the plane of the triangle. The distance between vertex $\mathrm{P}_{4}$ and the plane of the triangle is the length of the last vector, which is $\sqrt{4 / 3}$. Once the height is known we can calculate the volume of the tetrahedron

$$
V_{5}=6 \cdot h / 3=1 / 2 \cdot \sqrt{3} \cdot \sqrt{4 / 3} \cdot 1 / 3=1 / 3
$$

This values agrees with the volume calculated previously for $\mathrm{V}_{5}$.

In this case we could find by inspection the vector that is orthogonal to the edge chosen as the first vector in the plane. There are instances where it is not easy to find the second vector from looking at the figure. We can however make use of the idea that to find a vector orthogonal to another we can start with two arbitrary vectors in a plane and subtract from the first vector the projection of the second vector upon the first to end with a second vector orthogonal to the first. Were we looking for three orthogonal vectors we would orthogonalize the first two as indicated and then take a third arbitrary vector and subtract from it its projections upon the other two orthonormal vectors to find a third vector ortogonal to the first two. This last vector can then be normalized by dividing by its length. To illustrate this last method let us take as the two original vectors in the plane two of the edges: $P_{1} P_{2}$ and $P_{2} P_{3}$. The vector $P_{1} P_{2}$ was calculated before and normalized it turned out to be

$$
\mathrm{P}_{1} \mathrm{P}_{2}(\text { normalized })=(1 / \sqrt{2})(-1,1,0,0)
$$

The vector $\mathrm{P}_{2} \mathrm{P}_{3}$ is

$$
P_{2} P_{3}=(0,0,1,0)-(0,1,0,0)=(0,-1,1,0)
$$

The projection of $\mathrm{P}_{2} \mathrm{P}_{3}$ upon $\mathrm{P}_{1} \mathrm{P}_{2}$ (normalized) is

$$
(0,-1,1,0) \cdot(-1,1,0,0)(1 / \sqrt{2})=(1 / 2,-1 / 2,0,0)
$$

The second vector in the plane of the triangle, orthogonal to the first is

$$
(0,-1,1,0)-(1 / 2,-1 / 2,0,0)=(-1 / 2,-1 / 2,1,0)
$$

When this last vector is normalized we obtain as the second vector

$$
(1 / \sqrt{1.5})(-0.5,-0.5,1,0)
$$

Since this last vector is the same as the one obtained before, we need not continue since the results will be the same. We leave to the reader the independent verification of the fact that $V_{1}$ and $V_{2}$ are $\sqrt{76} / 6$

\section{Conclusion}

In this paper we have extended the Law of Cosines of the triangle to the tetrahedron and its multidimensional counterparts: the $n$-dimensional simplex. The Extended Law of Cosines has the following general structure

$$
\begin{gathered}
a^{2}=b^{2}+c^{2}+\ldots+z^{2}+2 b c \cos (b, c)+2 b d \cos \\
(b, d)+\ldots+2 y z \cos (y, z)
\end{gathered}
$$

Any set of vectors that when traversed form a closed cycle produce an Extended Law of Cosines, 
Any set of vectors that when traversed form a closed cycle produce an Extended Law of Cosines, therefore all closed polygons obey an extended Law of Cosines. By representing oriented plane surfaces with vectors orthogonal to the surfaces, it was shown that the representing vectors of tetrahedra and higher dimensional simplices form a closed cycle and therefore also obey an Extended Law of Cosines. By dividing polyhedra into simplices it can be shown that they also obey an Extended Law of Cosines. We provided proofs for tetrahedra and $n$-dimensional simplices of the validity of the Extended Law of Cosines using determinants as the tools to construct a mathematical induction. In the case of the tetrahedron we also used the alternative tool of the cross product between two three-dimensional vectors.

Although the cross product of two vectors exists oly in three dimensions, a useful cross product of $n-1$ vectors in $n$-dimensional space can be defined, with the property that the result is a vector that is orthogonal to all the vector factors. Such a vector product can be defined as follows

$\boldsymbol{x}_{1} \times \boldsymbol{x}_{2} \times \boldsymbol{x}_{3} \times \ldots \times \boldsymbol{x}_{n-1}=\left|\begin{array}{cccccc}x_{1}^{1} & x_{1}^{2} & x_{1}^{3} & \ldots & x_{1}^{n-1} & x_{1}^{n} \\ x_{2}^{1} & x_{2}^{2} & x_{2}^{3} & \ldots & x_{2}^{n-1} & x_{2}^{n} \\ \ldots & \ldots & \ldots & \ldots & \ldots & \ldots \\ x_{n-1}^{1} & x_{n-1}^{2} & x_{n-1}^{3} & \ldots & x_{n-1}^{n-1} & x_{n-1}^{n} \\ \boldsymbol{i} & \mathbf{i} & \boldsymbol{k} & \ldots & \boldsymbol{r} & \boldsymbol{s}\end{array}\right|$

where $\mathbf{i}, \boldsymbol{i}, \boldsymbol{k}, \ldots, \boldsymbol{r}, \boldsymbol{s}$ are unit vectors along $n$ orthogonal Cartesian axes and the $n-1$ vectors $\boldsymbol{x}_{1}, \boldsymbol{x}_{2}, \ldots, \boldsymbol{x}_{n-1}$ have components $\left(x_{1}^{1}, x_{1}^{2}, \ldots, x_{1}^{n}\right),($ $\left.x_{2}^{1}, x_{2}^{2} \ldots, x_{2}^{n}\right), \ldots,\left(x_{n-1}^{1} x_{n-1}^{2} \ldots, x_{n-1}^{n}\right)$ respectively. Finally we can define the magnitude of the resulting vector as the vector "hyperarea" of the base of an n-dimensional parallelotope; we can then introduce an additional vector $\boldsymbol{x}_{n}$ linearly independent from the first $n$ and the hypervolume of the parallelotope with edges meeting at a vertex given by the $n$ vectors is given by the dot product $\left(\boldsymbol{x}_{1} \times \boldsymbol{x}_{2} \times \boldsymbol{x}_{3} \times \ldots \times \boldsymbol{x}_{n-1}\right) . \boldsymbol{x}_{\boldsymbol{n}}$ which is a scalar that measures the volume of the parallelotope. The volume of the $n$-dimensional simplex having the same $n$ vectors meeting at one of the vertices is the same scalar multiplied by $1 / n$ ! This generalized cross product which is an extension of $\boldsymbol{a} \times \boldsymbol{b}$ defined for three-dimensional space has many of its properties. It is simultaneously orthogonal to all the factors for $n$ odd; a cyclic permutation of the factors leaves the result unaltered; and odd permutation results in a sign change, if the $n$ vectors are linearly dependent the result is zero, etc. All these properties can be derived from the properties of determinants. They can also be derived from purely geometrical concepts of n-dimensional space (Xambó, 1997).

We end this paper with a remark told to Felix Klein, the great German geometer, by the equally famous British mathematician Arthur Cayley (Klein, 1939): "If I had to give fifteen lectures on the whole of Mathematics, I would devote one of them to the theory of determinants." Among Numerical Analysts determinants have a bad name because, for instance, if Cramer's rule were used for the solution of dense sets of linear equations and the determinants were calculated using the formula based on even and odd permutations the amount of work would take even the fastest computers much more than thousands of years for even modest sets of fifty equations. However, for theoretical purposes, determinants are a great tool. Non other than Cayley says so

\section{References}

Bueche J.J. (1988). Física para Estudiantes de Ciencias e Ingeniería, Fourth Edition, Volume II. MC Graw-Hill/ Interamericana de México.

Cannon, Jr., R. H. (1967). Dynamics of Physical Systems. Mc Graw-Hill Book Company, New York.

Courant R. y Robbins H. (1941). What is Mathematics? Oxford University Press, London.

Dubrovin B., Nóvikov S. y Fomenko A. (1987). Geometría Moderna. Editorial Mir, Moscú.

Hague B. (1939). An Introduction to Vector Analysis. Methuen \& Co., Ltd., London.

Hildebrand F.B. (1952). Methods of Applied Mathematics. Prentice-Hall, Inc., Englewood Cliffs, NJ. 
Hillier F.S. y Lieberman G.J. (1974). Operations Research. (Second Edition). Holden Day, Inc., San Francisco.

Hoffmann B. (1966). About Vectors. Prentice-Hall, Inc., Englewood Cliffs, NJ.

Keedy M.L. y Nelson C.W. (1965). Geometry: A Modern Introduction. Addison-Wesley Publishing Company, Reading, MA.

Klein F. (1939). Elementary Mathematics from an Advanced Standpoint. Vol 2, Geometry. Dover Publications, Inc., New York.
Seely F.B. y Ensign N.E. (1948). Mecánica Analítica para Ingenieros. Unión Tipográfica Editorial Hispano-Americana, México.

Thomas, Jr. G.B. (1960). Calculus and Analytic Geometry. (Third Edition). Addison Wesley Publishing Company, Reading, MA.

Wagner H.M. (1969). Principles of Operations Research. Prentice-Hall, Inc. Englewood Cliffs, NJ.

Welchons A.M., Krickenberger W.R. y Pearsons H.R. (1965). Plane Geometry, Ginn and Company, Boston. Xambó-Descamps S. (1997). Geometría. Edicions UPC, Barcelona.

\section{Semblanza del autor}

Marco Antonio Murray-Lasso. Realizó la licenciatura en ingeniería mecánica-eléctrica en la Facultad de Ingeniería de la UNAM. El Instituto de Tecnología de Massachussetts (MIT) le otorgó los grados de maestro en ciencias en ingeniería eléctrica y doctor en ciencias cibernéticas. En México, ha laborado como investigador en el Instituto de Ingeniería y como profesor en la Facultad de Ingeniería (UNAM) durante 40 años; en el extranjero, ha sido asesor de la NASA en diseño de circuitos por computadora para aplicaciones espaciales, investigador en los Laboratorios Bell, así como profesor de la Universidad Case Western Reserve y Newark College of Engineering, en los Estados Unidos. Fue el presidente fundador de la Academia Nacional de Ingeniería de México; vicepresidente y presidente del Consejo de Academias de Ingeniería y Ciencias Tecnológicas (organización mundial con sede en Washington que agrupa las Academias Nacionales de Ingeniería) y secretario de la Academia Mexicana de Ciencias. Actualmente es jefe de la Unidad de Enseñanza Auxiliada por Computadora de la División de Estudios de Posgrado de la Facultad de Ingeniería de la UNAM, investigador nacional en ingeniería, vicepresidente de la Asociación de Informática en la Educación, consejero educativo del MIT y consultor de la UNESCO. 
